Martin Steskal

\title{
Rituelle Bestattungen im Prytaneion von Ephesos? Zu den Fundumständen der Artemis Ephesia-Statuen*
}

\section{Vorbemerkung}

1991/1992 veröffentlichte M. Donderer eine grundlegende Studie zur irreversiblen Deponierung von Großplastik in der Antike ${ }^{1}$. Wesentlicher Parameter einer solchen Deponierung sei, dass »die Gegenstände in der Antike mit Absicht vergraben worden sind«. Dies ließe sich vor allem aus der »außergewöhnlichen Lage« der Fundobjekte, die »auf eine möglichst unversehrte Deponierung abzielte«, erschließen. Diese intentionell deponierten Skulpturen wären daher meist besonders gut erhalten. Oftmals würde sich auch das Auffüllmaterial des Depots wesentlich vom umgebenden Erdreich des Fundplatzes unterscheiden und somit einen künstlichen Eingriff anzeigen. Die Gründe für irreversible Deponierungen lägen laut M. Donderer vorwiegend im religiösen Bereich²: „Wo es in den antiken Kulturen Kultstatuen gab, waren sie unantastbar. ... Wurden sie - meist infolge einer Beschädigung - durch neue ersetzt, so vergrub man offenbar die alten in der Regel im Heiligtum.«

Im Folgenden sollen die Fundsituationen von vier Skulpturen, und zwar der Artemis Ephesia-Statuen aus dem Prytaneion von Ephesos ${ }^{3}$ (Abb. 1), die seit ihrem Auffinden in den 1950er Jahren immer wieder mit einer irreversiblen Deponierung - konkret mit einer `kultischen Bestattung $<$ - in Verbindung gebracht werden, evaluiert werden. Inwieweit bei diesen Beispielen eine kultische oder rituelle Bestattung stattgefunden hat oder eine solche heute zu belegen ist, soll durch eine Analyse der Fundsituation geklärt werden.

\footnotetext{
* Mein Dank gilt der stellvertretenden Grabungsleiterin von Ephesos, S. Ladstätter, sowie dem ehemaligen Grabungsleiter, F. Krinzinger, für die Unterstützung meiner Arbeiten am Prytaneion. Für wertvolle Hinweise und eine fruchtbare Diskussion sei insbesondere M. Aurenhammer gedankt. Für die Durchsicht des Manuskripts danke ich B. Horejs und S. Ladstätter. Die Arbeiten am Prytaneion von Ephesos werden vom Fonds zur Förderung der wissenschaftlichen Forschung finanziert (FWF-Projekt P19257G02). - Zusätzlich zu den vom Österreichischen Archäologischen Institut empfohlenen Abkürzungen <http://www.oeai.at/ publik/autoren.html> werden in diesem Beitrag verwendet:

Alzinger 1962 W. Alzinger, Die Stadt des siebenten Weltwunders. Die Wiederentdeckung von Ephesos (Wien 1962).

Alzinger 1972-1975 W. Alzinger, Das Regierungsviertel, ÖJh 50, 1972-1975, Beibl. 229-300.

Donderer 1991/1992 M. Donderer, Irreversible Deponierung von Großplastik bei Griechen, Etruskern und Römern, ÖJh 61, 1991/1992, Beibl. 193-276.

Friesinger - Krinzinger $1999 \quad$ H. Friesinger - F. Krinzinger (Hrsg.), 100 Jahre Österreichische Forschungen in Ephesos. Akten des Symposions Wien 1995, AForsch 1 = DenkschrWien 260 (Wien 1999).

IvE Inschriften griechischer Städte aus Kleinasien 11, 1 (Bonn 1979) - 17, 4 (Bonn 1984) = Ephesos: IvE Ia-VIII 2.

Miltner 1957 F. Miltner, Bericht über die Ausgrabungen in Ephesos 1956, TAD 7, 1957, 20-25.

Miltner 1958a $\quad$ F. Miltner, Die neuen Artemisstatuen aus Ephesos, Anatolia 3, 1958, 21-34.

Miltner 1958b F. Miltner, Ephesos. Stadt der Artemis und des Johannes (Wien 1958).

Miltner 1959 F. Miltner, XXII. Vorläufiger Bericht über die Ausgrabungen in Ephesos, ÖJh 44, 1959, Beibl. $289-312$.

${ }^{1}$ Donderer 1991/1992, 193-276; vgl. auch M. Donderer, Irreversible Deponierung von Architekturteilen bei Griechen, Etruskern und Römern, ÖJh 62, 1993, Beibl. 93-134.

2 Donderer 1991/1992, 203.

${ }^{3}$ Zum Gebäude: F. Miltner, XXI. Vorläufiger Bericht über die Ausgrabungen in Ephesos, ÖJh 43, 1956-1958, Beibl. 27-36; F. Miltner, Ergebnisse der österreichischen Ausgrabungen in Ephesos im Jahre 1956, AnzWien 94, 1957, 23-25; Miltner 1959, 289-312; F. Eichler, Die österreichischen Ausgrabungen in Ephesos im Jahre 1960, AnzWien 98, 1961, 66-69; F. Eichler, Die
} 


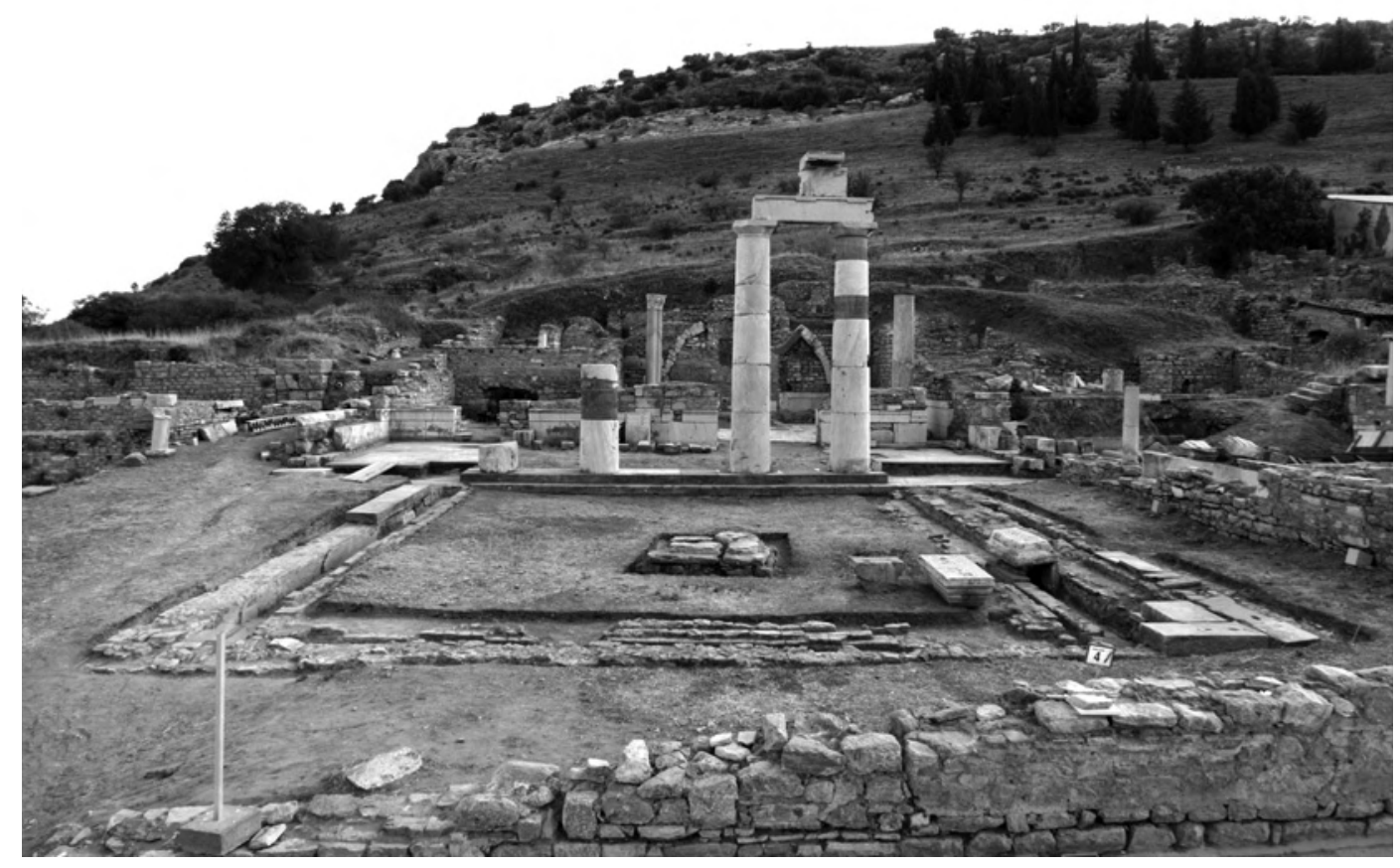

1 Ephesos. Prytaneion von Süden

\section{Fundsituation der Artemis Ephesia-Statuen}

Das Kultbild der Artemis Ephesia ist vor allem aus Nachbildungen der römischen Kaiserzeit bekannt. Gleich mehrere Varianten dieses Statuentypus unterschiedlichen Erhaltungszustands konnte F. Miltner im Zuge seiner Freilegung des Prytaneions von Ephesos in den 1950er Jahren ans Tageslicht bringen (Abb. 2) ${ }^{4}$ : Es handelt sich dabei um die Statuen der `Schönen Artemis` (Efes Müzesi Selçuk Inv. 718; Abb. 3. 4), der 〉Großen Artemis` (Efes Müzesi Selçuk Inv. 712; Abb. 6. 7) und der `Kleinen Artemis` (Efes Müzesi Selçuk Inv. 717; Abb. 5). Von letztgenannter Statue, die offenbar in Serie produziert worden war, entdeckte er das Fragment eines weiteren Exemplars (Depot des Efes Müzesi Selçuk, Fundnr. 231/56). Die Funde tätigte er in kurzen Abständen zwischen dem 18. September und 3. Oktober 1956.

Die exzeptionellen Skulpturen wurden seit ihrer Auffindung bis zum heutigen Tag einer kaum noch zu überblickenden Zahl von kulturhistorischen und stilkritischen Studien unterzogen ${ }^{5}$. Eine dieser Statuen,

österreichischen Ausgrabungen in Ephesos im Jahre 1961, AnzWien 99, 1962, 38-40; F. Eichler, Die österreichischen Ausgrabungen in Ephesos im Jahre 1962, AnzWien 100, 1963, 46; F. Eichler, Die österreichischen Ausgrabungen in Ephesos im Jahre 1963, AnzWien 101, 1964, 40-41; Alzinger 1972-195, 233-249; W. Alzinger, Augusteische Architektur in Ephesos, SoSchrÖAI 16 (Wien 1974) 51-55; S. G. Miller, The Prytaneion, its Function and Architectural Form (Berkeley 1978) 98-109; D. Knibbe, Der Staatsmarkt. Die Inschriften des Prytaneions, FiE 9, 1, 1 (Wien 1981); H. Thür, Wie römisch ist der sog. Staatsmarkt in Ephesos?, in: M. Meyer (Hrsg.), Neue Zeiten - Neue Sitten. Zu Rezeption und Integration römischen und italischen Kulturguts in Kleinasien. Akten des Internationalen Kolloquiums in Wien 2005, WForsch 12 (Wien 2007) 77-90; A. Bammer, Zur Dekonstruktion römischer Architektur. Studien zur Architektur im Nordbereich der sog. oberen Agora von Ephesos, Anatolia Antiqua 16, 2008, 165-180.

${ }^{4}$ Miltner 1959, 305-309; Miltner 1958a, 21-34; Miltner 1957, 23 f.

${ }_{5}$ Auswahl: Miltner 1959, 305-309; RE Suppl. XII (1970) 323-328 s. v. Ephesos: C. Numismatischer Teil (St. Karwiese); R. Fleischer, Artemis von Ephesos und verwandte Kultstatuen aus Anatolien und Syrien, EPRO 35 (Leiden 1973) 14 f. 46-137; E. Heinzel, Zum Kult der Artemis von Ephesos, ÖJh 50, 1972-1975, 243-251; R. Fleischer, Artemis Ephesia, in: A. Bammer R. Fleischer - D. Knibbe, Führer durch das Archäologische Museum in Selçuk-Ephesos (Wien 1974) 18-21 mit weiterer Lit.; R. Fleischer, Artemis von Ephesos und verwandte Kultstatuen aus Anatolien und Syrien, in: S. Şahin - E. Schwertheim J. Wagner (Hrsg.), Studien zur Religion und Kultur Kleinasiens. Festschrift Friedrich Karl Dörner, EPRO 66, 1 (Leiden 1978) 324-358; R. Fleischer, Die Kultstatue der Artemis von Ephesos und verwandte Götterbilder, in: W. Seipel (Hrsg)., Das Artemision von Ephesos. Heiliger Platz einer Göttin (Wien 2008) 25-41; E. Lessing - W. Oberleitner, Ephesos. Weltstadt der Antike 


\section{PRYTANEION}

Fundpunkte Artemis Ephesia-Statuen

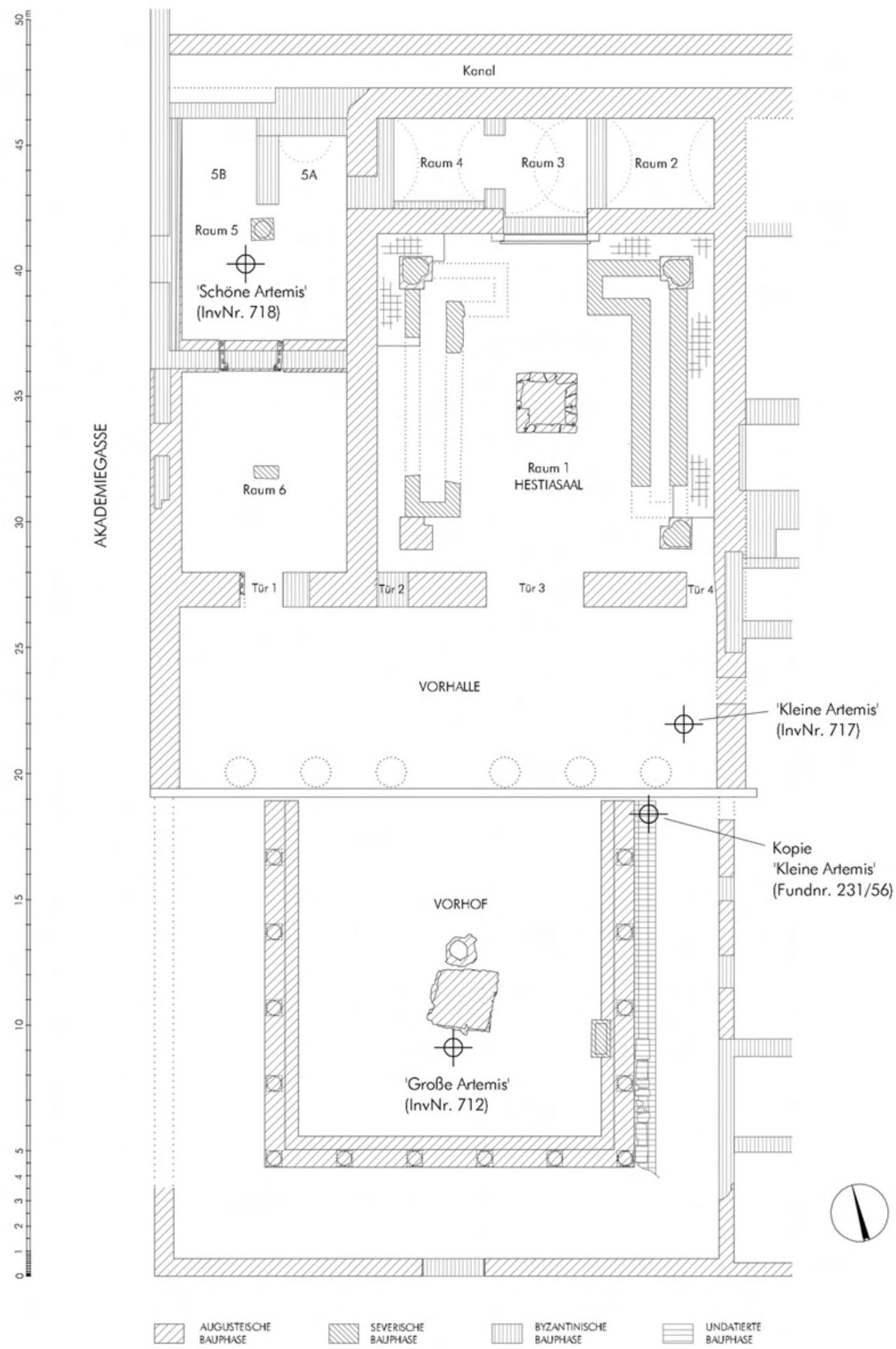

2 Prytaneion. Generalisierter Grundriss mit Fundlagen der Artemis Ephesia-Statuen (M. $1: 250$ ) 
und zwar die >Schöne Artemis $\measuredangle$, versah der Ausgräber mit dem Prädikat einer $>$ kultischen Bestattung $\iota^{6}: »$ Der wichtigste Fund aber stellte sich im südlichen der beiden Westräume (Raum 5, Anm. d. Verf.) ein 7 . Hier lag wenig über dem Boden auf dem Rücken auch eine Statue der Artemis Ephesia, an welcher noch geringe Reste einstiger Vergoldung vorhanden waren. Es fehlen zwar der Kopfaufsatz, Teile der Säulchen und manches von den beiden Hirschkühen; all das muß verlorengegangen sein, als die Statue noch aufgestellt war. Denn an die Fundstelle ist sie nicht durch Sturz gekommen, sondern eindeutig durch Bestattung; das ergibt sich nicht nur aus der Lage, sondern vor allem aus dem sie umgebenden, nur mit ganz wenig Ziegelbröckchen durchmischten, auffällig schwarzen Erdreich. Wer sie bestattet hat, ist nicht feststellbar. Man wird am ehesten an ihre letzten Anhänger denken, die das vielleicht umgeworfene Bild hier bargen und alle noch auffindbaren Stücke der zerbrochenen Säulchen und Hirschkühe sorgsam dazu legten, während der Bau von den Christen im heiligen Eifer niedergerissen wurde.« Die zerbrochenen sog. Säulchen und Hirschkühe wären weiters auf die Ungeschicklichkeit der Bestatter beim Herunternehmen der Statue von ihrem Sockel zurückzuführen.

Dieser Sichtweise - und zwar der rituellen Bestattung dieser Skulptur - schloss sich eine große Zahl Forscherinnen und Forscher $a^{8}{ }^{8}$. Und obwohl F. Miltner dezidiert nur die >Schöne Artemis` mit >kultischer

(Wien 1978) 188. 239-241; G. Seiterle, Artemis, die große Göttin von Ephesos. Eine neue Deutung der Vielbrüstigkeit eröffnet einen Zugang zum bisher unbekannten Kult der Göttin, AW 10/3, 1979, 3-16; R. Fleischer, Neues zu kleinasiatischen Kultstatuen, AA 1983, 81-93; LIMC II 1 (1984) 755-763 s. v. Artemis Ephesia (R. Fleischer); L. R. LiDonnici, The Images of Artemis Ephesia and Greco-Roman Worship. A Reconsideration, HarvTheolR 85, 1992, 389-415; A. Bammer - U. Muss, Das Artemision von Ephesos. Das Weltwunder Ioniens in archaischer und klassischer Zeit (Mainz 1996) 71-78; W. Burkert, Die Artemis der Epheser: Wirkungsmacht und Gestalt einer Großen Göttin, in: Friesinger - Krinzinger 1999, 59-70; R. Fleischer, Neues zum Kultbild der Artemis von Ephesos, in: Friesinger - Krinzinger 1999, 605-609; U. Muss, Zur Dialektik von Kultstatue und Statuetten im Artemision von Ephesos, in: Friesinger - Krinzinger 1999, 597-603; L. Portefaix, The Image of Artemis Ephesia - A Symbolic Configuration Related to her Mysteries?, in: Friesinger - Krinzinger 1999, 611-617; St. Karwiese, Artemis Ephesia `Sebasteia<: Ein Entzifferungsbeitrag, in: P. Scherrer - H. Taeuber - H. Thür (Hrsg.), Steine und Wege. Festschrift Dieter Knibbe, SoSchrÖAI 32 (Wien 1999) 61-75; R. Fleischer, Eine silberne Hand der Artemis von Ephesos im Archäologischen Museum der Universität Münster, Boreas 23/24, 2000/2001, 191-194; S. P. Morris, The Prehistoric Background of Artemis Ephesia: A Solution to the Enigma of her >Breasts`?, in: U. Muss (Hrsg.), Der Kosmos der Artemis von Ephesos, SoSchrÖAI 37 (Wien 2001) 135-151; K. Radner, Kompositstatuen vom Typus der Ephesia aus dem vorkroisoszeitlichen Heiligtum. Zur Herstellung und Pflege von Götterstatuen im östlichen Mittelmeerraum und im Vorderen Orient im frühen ersten Jahrtausend, in: Muss a. O. 233-263; R. Fleischer, Die Amazonen und das Asyl des Artemisions von Ephesos, JdI 117, 2002, 185-216 bes. 208-215; S. Szidat, Die `Buckel< der Artemis Ephesia. Zur Bedeutung des Motivs und seinen ikonographischen Vorläufern, JdI 119, 2004, 83-129. - Zum Typus s. auch: H. Thiersch, Artemis Ephesia. Eine archäologische Untersuchung, AbhGöttingen 3, 12 (Berlin 1935).

${ }^{6}$ Miltner 1959, 308 f.; vgl. auch Miltner 1958b, 97-104. bes. 104: »Darf die Statue ob ihres künstlerischen Wertes zu den hervorragendsten Funden der ephesischen Grabung gerechnet werden, so ist sie nicht minder bedeutungsvoll deshalb, weil sie eben hier in dem Nebenraum des Hestiaheiligtumes offenkundig mit Absicht bestattet worden ist. Der Grabungsbefund sagt freilich nichts darüber aus, wer die Statue der hohen Göttin so sorgsam hier in die Erde gebettet hat. ... Doch unabhängig davon lehrt die Bestattung des Bildes, ob auf diese oder die andere Weise, daß auch in dem Jahrhundert, da das Christentum seine Hauptkirche in die Mauern der einstigen Schule der hohen heidnischen Geistigkeit hineinbauen konnte, doch noch scheue Herzen dem Bilde der Göttin - sei es als verehrende Anhänger, sei es in zagender Furcht - verbunden waren.«; Miltner 1958a, 32 f.: »Bei der gewaltsamen Zerstörung dieses Ersatzsitzungssaales, welche mit guten Gründen an der Wende vom 4. zum 5. Jh. n. Chr. angesetzt werden darf, ist die Statue aber vermutlich von besonderen Verehrern vor den Zerstörern, in denen wir Christen zu erkennen haben, eben in den Nebenraum gebracht und hier vergraben worden. Denn das liess die Ausgrabung deutlich werden, dass die Statue nicht etwa beim Einsturz dieses Nebenraumes von dem Schutt begraben wurde, sondern dass sie in diesem Raum, solange dessen Wände noch standen, sorgfältig hier auf eingebrachter Erde niedergelegt und mit ebensolcher Erde zugedeckt, also bestattet worden ist.«; Miltner 1957, 24.

7 Tatsächlich handelt es sich um den nördlichen der beiden Westräume, also Raum 5, wie eine Überprüfung der Grabungstagebücher und der historischen Photographien ergab. Für den Hinweis danke ich M. Aurenhammer.

${ }^{8}$ Alzinger 1962, 224; Lessing - Oberleitner (Anm. 5) 188. 239-241; Seiterle (Anm. 5) 7; Donderer 1991/1992, 248 f. Nr. 35 ; D. Knibbe, Via Sacra Ephesiaca: New Aspects of the Cult of Artemis Ephesia, in: H. Koester (Hrsg.), Ephesos. Metropolis of Asia. An Interdisciplinary Approach to its Archaeology, Religion, and Culture, Harvard Theological Studies 41 (Valley Forge 1995) 146 Anm. 8; W. Elliger, Ephesos. Geschichte einer antiken Weltstadt ${ }^{2}$ (Stuttgart 1992) 62 f.; T. Wohlers-Scharf, Die Forschungsgeschichte von Ephesos. Entdeckungen, Grabungen und Persönlichkeiten, Europäische Hochschulschriften 38, 54 ${ }^{2}$ (Frankfurt 1996) 200 f.; D. Knibbe, Ephesus. E $\Phi E \Sigma O \Sigma$. Geschichte einer bedeutenden antiken Stadt und Portrait einer modernen Großgrabung im 102. Jahr der Wiederkehr des Beginnes österreichischer Forschungen (1895-1997) (Frankfurt 1998) 29; Burkert (Anm. 5) 59; Portefaix (Anm. 5) 617; W. Alzinger, Das Zentrum der lysimachischen Stadt, in: Friesinger - Krinzinger 1999, 390; St. Karwiese, Die Münzfunde aus dem sog. Sockelbau der Basilica von Ephesos, in: H. Emmerig (Hrsg.), Vindobona docet. 40 Jahre Institut für Numismatik und Geldgeschichte der Universität Wien 1965-2005 (Wien 2005) 181 Anm. 6; vgl. auch IvE 1351. 


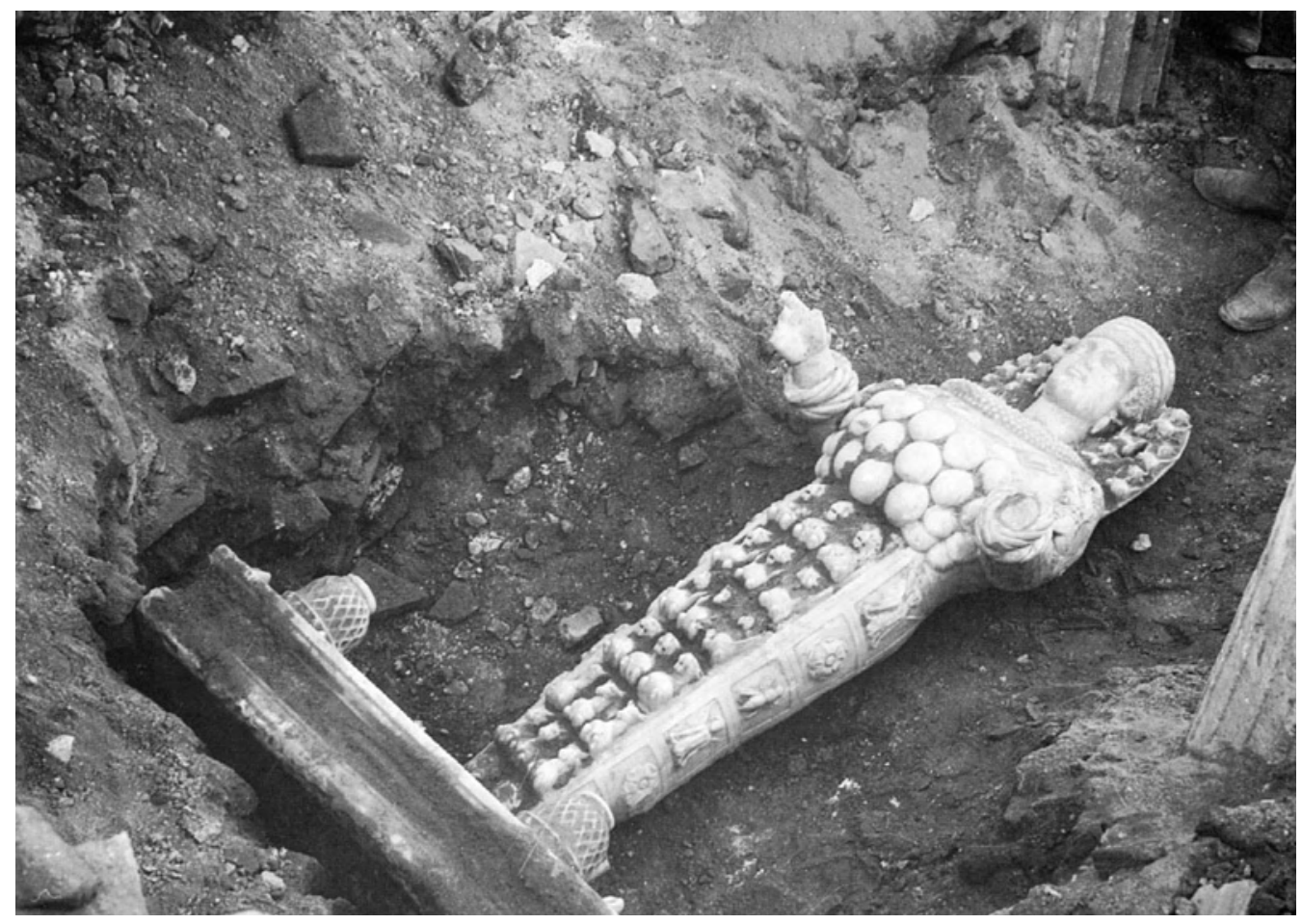

3 >Schöne Artemis` in Fundlage (Raum 5)

Bestattung in Verbindung bringt, wird dieses Phänomen von mehreren Forschern auch auf die anderen im Prytaneion gefundenen Artemis Ephesia-Statuen übertragen, ohne dass während der Ausgrabungen entsprechende Beobachtungen gemacht oder später vom Ausgräber veröffentlicht worden wären ${ }^{9}$. Dies belegen sowohl die Einträge in das Originalgrabungstagebuch ${ }^{10}$ als auch die Publikation der Fundstücke:

1) >Kleine Artemis` (2 Exemplare: Efes Müzesi Inv. 717 und Fundnr. 231/56)

F. Miltner, Tagebucheintrag vom 28. 9. 1956: »Im Osteck der Vorhalle, wo an einer Stelle der Mosaikboden erreicht wird, wird abermals eine Kultstatue der Artemis gefunden, jedoch kopflos und ohne Basis: etwa halbe Lebensgrösse, Marmor der auch schon in Ephesos üblich.«

F. Miltner, Tagebucheintrag vom 13. 10. 1956: »... im Nordosteck der Halle Fussteil einer weiteren Artemis Ephesiastatue und zwar eines zweiten Exemplares der zweiten."

Miltner 1959, 307 f.: »Am Ostende der Vorhalle fand sich eine unterlebensgroße Statue der Artemis; Kopf und Hände fehlen und waren einst angedübelt. Es handelt sich um die mittelmäßige Arbeit einer Werkstatt, die diese Statue offenbar in Serie herstellte, da noch der Fußteil eines nach Marmor und Arbeit völlig gleichen Stückes wenig westlich davon vor der Vorhalle gefunden wurde.«

2) >Große Artemis`(1 Exemplar: Efes Müzesi Inv. 712)

F. Miltner, Tagebucheintrag vom 2./3. 10. 1956: »In der Vorhalle wird nach Süden gegraben und von der Strassenrückwand nach Norden. Dabei wird im Südteil der Torso einer dritten Artemisstatue gefunden, westlich von welchem eine weitere Statue liegt.«

\footnotetext{
${ }^{9}$ M. Büyükkolanc1 - Ü. Yüğrük, Prytaneion, in: P. Scherrer (Hrsg.), Ephesos. Der neue Führer (Wien 1995) 86-88; St. Karwiese, Groß ist die Artemis von Ephesos. Die Geschichte einer der großen Städte der Antike (Wien 1995) 131 f.; D. Knibbe, Via Sacra Ephesiaca, in: Friesinger - Krinzinger 1999, 451; P. Scherrer, The City of Ephesos from the Roman Period to Late Antiquity, in: Koester (Anm. 8) 19; H. Thür, Das spätantike Ephesos. Aspekte zur Frage der Christianisierung des Stadtbildes, in: G. Brands H.-G. Severin, Die spätantike Stadt und ihre Christianisierung. Symposium vom 14. bis 16. Februar 2000 in Halle/Saale (Wiesbaden 2003) 264. - Indifferent: C. Foss, Ephesus after Antiquity: A late Antique, Byzantine and Turkish City (Cambridge 1979) 80 .

${ }^{10}$ Die zitierten Grabungstagebücher werden im Archiv des ÖAI verwahrt und können dort eingesehen werden.
} 


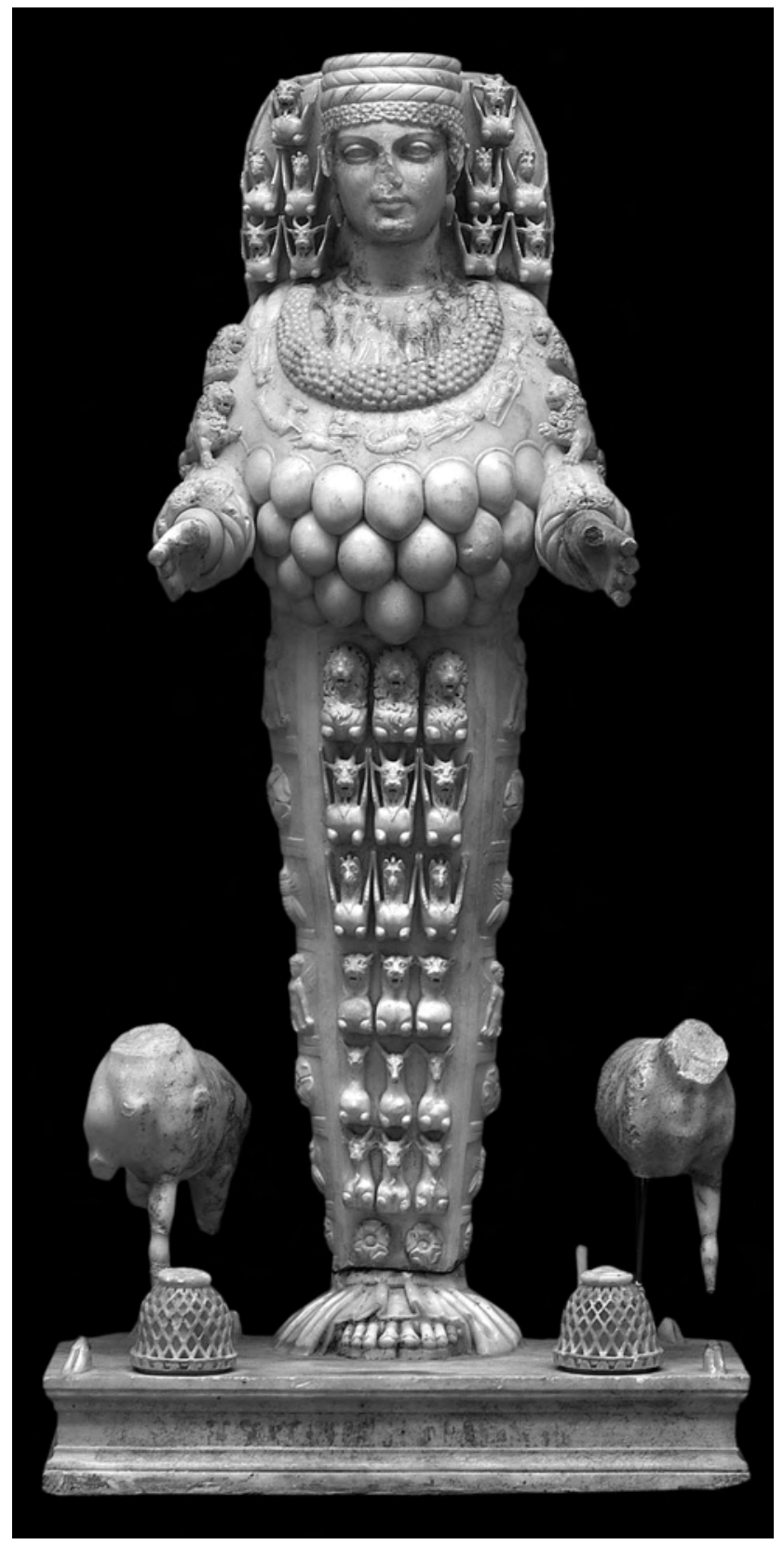

4 `Schöne Artemis` (Efes Müzesi Inv. 718)

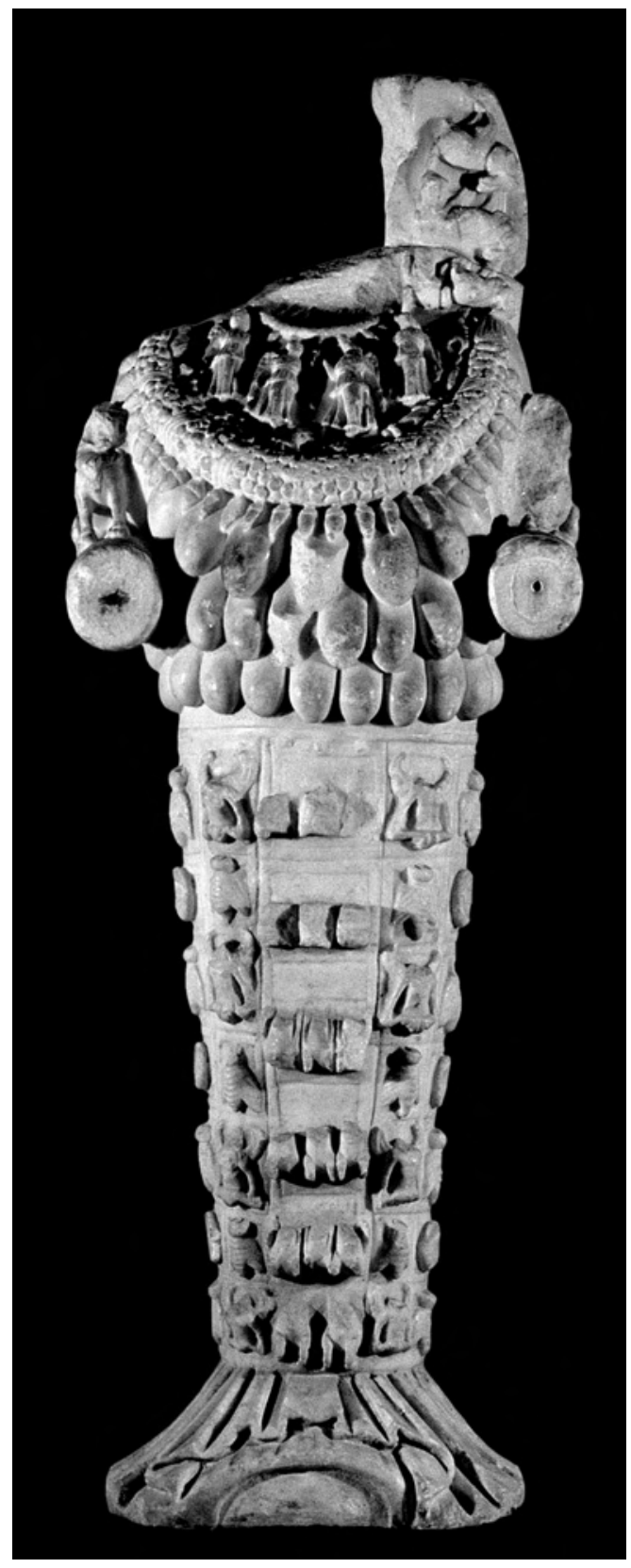

5 >Kleine Artemis` (Efes Müzesi Inv. 717)

F. Miltner, Tagebucheintrag vom 4. 10. 1956: »Was am Vortage als eine zweite Statue angesehen wurde, erweist sich als Kopf mit dem vollständigen Aufsatz der gestern gehobenen Statue, welche ohne die fehlende Fußpartie bereits 2,70 m hoch ist. Sehr gute Arbeit."

Miltner 1959, 305-307: »Auf dem Vorplatz des Herdsaales wurde südlich des Postamentsockels eine Statue der Artemis Ephesia von etwa zweifacher Lebensgröße gefunden, die auf dem Gesicht lag und durch den Sturz in zwei sauber anpassende Stücke zerbrochen war. Diese Statue, der nur das Fußstück fehlt und die eine Kopie aus dem Ende des 1. Jhs. n. Chr. sein dürfte, ist vor allem wegen des vollständig erhaltenen Kopfaufsatzes mit den verschiedenen Tempeldarstellungen von maßgebender Bedeutung. Man wird sich den Sockel, auf dem sie aufgestellt war, nicht allzu niedrig vorstellen, so daß der Gesamteindruck der Statue, die 


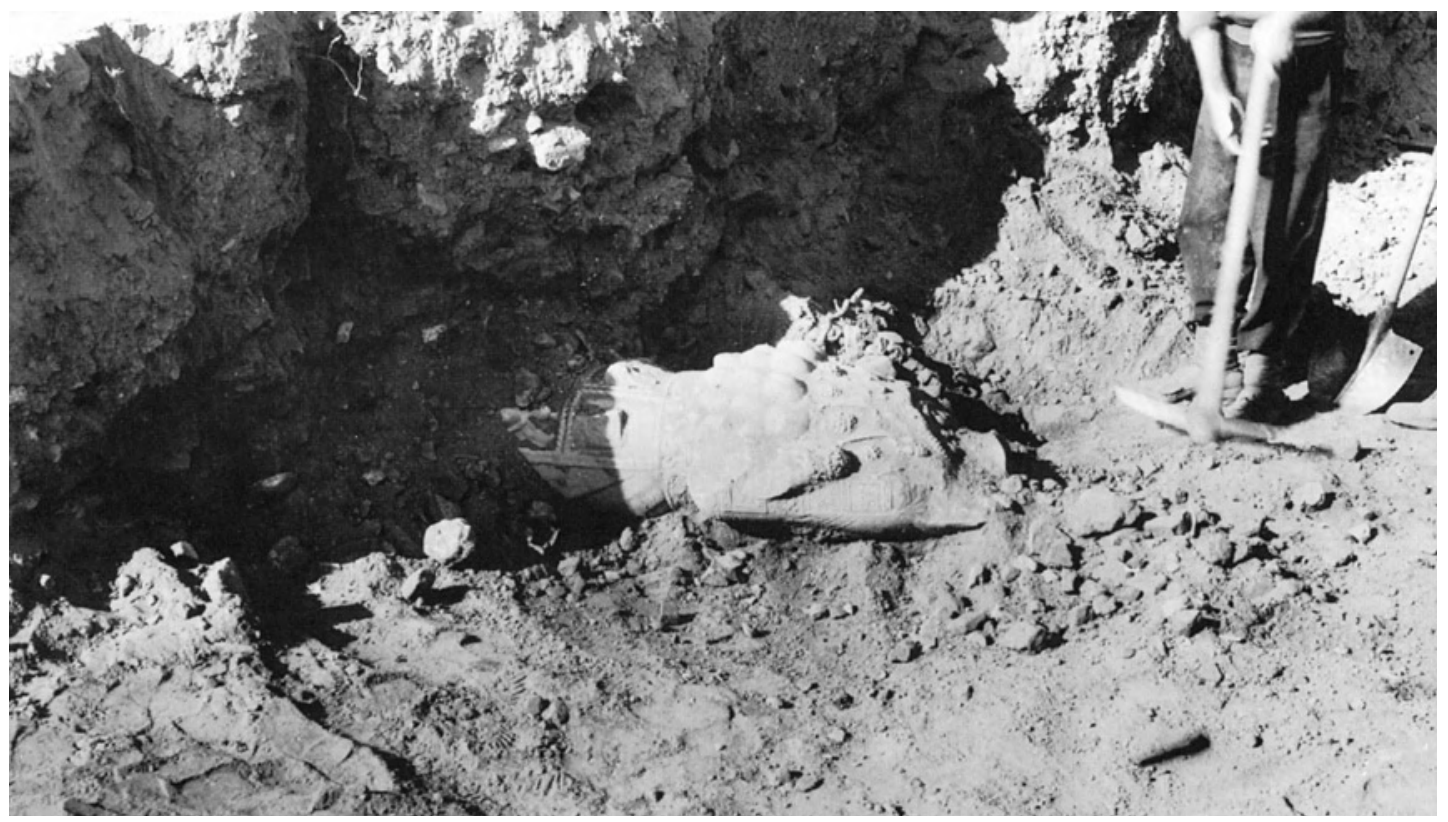

6 >Große Artemis` in Fundlage (Vorhof)

der Fallage gemäß nach Süden geblickt hat, in der Umrahmung der jonischen Säulenhalle gewaltig gewesen sein $\mathrm{mu}$. $\ll^{11}$

Während es also für eine >kultische Bestattung der im Prytaneion gefundenen Artemis Ephesia-Statuen vom Typus der 〉Kleinen Artemis` und 〉Großen Artemis` keinen positiven Beleg gibt und im Zuge der Ausgrabung keine diesbezüglichen Beobachtungen gemacht wurden, gilt es zu überprüfen, inwieweit dies auf die `Schöne Artemis` zutrifft. Auch hier sei zunächst das Originalgrabungstagebuch bemüht:

3) >Schöne Artemis` (1 Exemplar: Efes Müzesi Inv. 718)

F. Miltner, Tagebucheintrag vom 18. 9. 1956: »In dem Raum, in welchem die vielen Näpfchen und Freskenreste gefunden worden waren (Raum 5, Anm. d. Verf.), wird auf dem Boden in Rückenlage eine aus durchschimmernden glänzend poliertem Marmor gefertigte Kultstatue der Artemis gefunden. Auf dem Gesicht und der Brust noch beträchtliche Spuren der einstigen Vergoldung. Es muss erst die weitere Freilegung zeigen, ob die Statue hierher gestürzt ist oder aber hereingelegt wurde. Die beiden Rehe sind weggebrochen, doch lassen einige schon aufgefundene Fragmente hoffen, dass mehr oder weniger die gesamte Statue, welche eine Arbeit des 2. Jhs. sein dürfte, zusammenkommen wird. Die Statue ist bis auf eine geringfügige Beschädigung der Nase und, von den abgebrochenen Händen abgesehen, vollständig. Knapp über dem niederen Standsockel ist sie in zwei Teile gebrochen.«

Durch die Besonderheit des Fundes verpflichtet, notiert F. Miltner - als eine der wenigen Ausnahmen folgende schichtenspezifische Beobachtung in das Tagebuch ${ }^{12}$ : »Im Westraum (Raum 5, Anm. d. Verf.) lässt Keramik annehmen, dass das Artemisniveau etwa im 3. Jh. entstanden ist; beim Tiefergraben unter dieses Niveau treten Lampen des 1. Jhs. auf.« Da er die Fundstücke in der Folge weder inventarisiert noch dokumentiert, sind diese Datierungsansätze heute nicht mehr zu überprüfen. Der von Miltner im Grabungstagebuch als »Artemisniveau« bezeichnete Nutzungshorizont, den er ca. $30 \mathrm{~cm}$ über dem ursprünglichen Lauf-

\footnotetext{
${ }^{11}$ Gegen eine ungestörte Sturzlage der >Großen Artemis` äußert sich später Alzinger 1972-1975, 242, der im zentralen Fundament des Vorhofes eher die Unterkonstruktion eines Altars sehen wollte: »Die große Artemisstatue wurde an dieser Stelle gefunden, übrigens ohne Füße, was eher gegen als für eine ungestörte Sturzlage spricht.« Diese Sicht der Dinge relativiert er letztlich wieder. Vgl. W. Alzinger, Das Zentrum der lysimachischen Stadt, in: Friesinger - Krinzinger 1999, 390: »Der Platz vor dem Prytaneion war wohl für die im Frühherbst 1956 gefundene kolossale Marmorstatue der Artemis vorgesehen; ...«

${ }^{12}$ F. Miltner, Tagebucheintrag vom 28. 9. 1956.
} 
niveau lokalisiert ${ }^{13}$, führt allerdings zu erheblichen Ungereimtheiten hinsichtlich der Deponierungssituation der `Schönen Artemis`. So wurden weitere Fragmente der Statue in Schichten unter diesem Stratum gefunden $^{14}: »$ Am Hestiasaal wird im Raum 6 die ursprüngliche Südwand freigemacht, in der annähernd axial eine breite Tür nach Süden sich öffnet; die ursprüngliche Mauer biegt im Westen nach Norden um; im Schutt werden, und zwar in den untersten Schichten, weitere Fragmente der ersten Artemis gefunden.« Ein weiteres Fragment der >Schönen Artemis` findet W. Alzinger 1961 in Raum 6 beim »Abgraben auf das Niveau des Hestiasaales « und somit wiederum unter dem $>$ Artemisniveau ${ }^{15}$. Teile der Statue wären folglich vergraben gewesen, während der Großteil der Statue auf (sic!) dem Nutzungshorizont gelegen wäre. Andere Teile, wie etwa der Kopfaufsatz, fehlen überhaupt.

Der Großteil der Statue war also nicht vergraben oder gar bestattet, sondern kam - aus Gründen, die uns nicht bekannt sind, - auf dem >Artemisniveau< zu liegen. Vergraben und bestattet hätte die Statue nur von einem noch jüngeren und höher gelegenen Nutzungsniveau werden können. Bei der Anlage der byzantinischen Nutzungsniveaus auf den Zerstörungsstraten des Prytaneions war das Gebäude allerdings schon devastiert, sein Aufgehendes großteils eingerissen und die Räume der Anlage mit bis zu $2 \mathrm{~m}$ hohem Schutt bedeckt; auch die Statue wird zu diesem Zeitpunkt kaum noch sichtbar an ihrem ursprünglichen Platz gestanden sein. Aus der Fundlage ist somit kein wie auch immer gearteter Beleg für eine `kultische Bestattung`abzulesen. Auch zum Zeitpunkt der Auffindung ist der Ausgräber - wie aus dem Tagebucheintrag hervorgeht - über die Form der Deponierung im Unklaren. Eine gesicherte Beurteilung des Befundes wäre nur dann möglich gewesen, wenn die Grabungsarbeiten unter permanenter archäologischer Aufsicht vonstatten gegangen wären, was aber keineswegs der Fall gewesen zu sein scheint, wie eine Beschreibung der Fundumstände von W. Alzinger ergibt ${ }^{16}$. Eine profunde Analyse der Stratigraphie kann unter diesen Voraussetzungen und einer erst nachträglichen Autopsie der Fundstelle nicht erfolgt sein, wie der spärliche Dokumentationsgrad der Arbeiten F. Miltners in den 1950er Jahren belegt ${ }^{17}$. Von einer gesicherten >Bestattung « des Kultbildes kann aufgrund der Fundlage und des Befundes somit keine Rede sein, im Gegenteil: Sie ist rein spekulativ. Nicht klären kann Miltner ferner, warum die »letzten Anhänger« der Göttin nur eine der vier im Prytaneion aufgestellten Artemis Ephesia-Statuen bestatteten und die anderen drei in ihrer Sturz- oder Deponierungslage zurückließen.

Für die Bestattung der \Schönen Artemis〈 macht F. Miltner die »letzten Anhänger« der Göttin verantwortlich, die die Statue vor der Zerstörungswut der Christen retten wollten ${ }^{18}$. Namentlich schreibt er der Christin Scholastikia die Zerstörung des Gebäudes zu ${ }^{19}$ : Es ist aber auch zu erwägen, ob nicht Scholastikia dieses Heiligtum in ihrem heiligen Eifer für die Lehre des Evangeliums alles, was heidnisch war, zu verdammen, selbst zerstört hat.« Dem nicht genug, macht Miltner Scholastikia auch für das Umstürzen der im

${ }^{13}$ F. Miltner, Tagebucheintrag vom 2./3. 10. 1956: »Am Hestiasaal geht die Ausräumung weiter. Die Mauern am Artemisraum haben ihr ursprüngliches Bodenniveau etwa $30 \mathrm{~cm}$ unter dem Artemisniveau. « Für die tatsächliche Existenz des >Artemisniveaus $<-$ eine zeichnerische Dokumentation liegt nicht vor - sprechen nachträgliche Notizen des damaligen Mitarbeiters, W. Alzinger, in sein Tagebuch vom 20. und 24. 9. 1956, wonach die heute noch in situ in Raum 5 stehende Säule nicht auf dem Fußboden stehen, sondern tiefer gehen würde. Südlich dieser Säule und zwischen zwei weiteren kannelierten Säulen, die auf dem >Artemisniveau< gestanden wären, hätte sich die Statue befunden. $\mathrm{Zu}$ einer >kultischen Bestattungく äußert er sich nicht.

${ }_{14}$ F. Miltner, Tagebucheintrag vom 26. 10. 1956.

15 W. Alzinger, Tagebucheintrag vom 9. 8. 1961.

${ }^{16}$ So wird der Fund der \Schönen Artemis`von Alzinger 1962, 224 - im Fundjahr 1956 bereits Teilnehmer der Ausgrabungen in Ephesos - nachträglich in seiner populärwissenschaftlichen Ephesos-Publikation wie folgt beschrieben: »Es war ein sonniger, sehr warmer Nachmittag. Miltner war gerade mit der Aufnahme von Inschriften bei der Scholastikiatherme beschäftigt, als plötzlich einer der türkischen Grabungswächter aufgeregt auf ihn zustürzte: >Profesor bey, Profesor bey, güzel bir heykel bulduk, bütün altın! (Herr Professor, Herr Professor, wir haben eine schöne Statue gefunden, ganz aus Gold!) Nun, dachte Miltner, ganz aus Gold wird sie schon nicht sein, aber etwas Besonderes wird sicher dahinterstecken. So schnell er konnte, eilte er also zum Rathaus. Und tatsächlich bot sich ihm ein wunderbarer Anblick! Eine schöne Statue der Artemis Ephesia, sehr gut erhalten, etwas unterlebensgroß, lag auf dem Rücken im Erdreich, so, als hätte man sie behutsam eingebettet."

${ }^{17}$ Auch auf der Photographie der `Schönen Artemis` in Fundlage (Abb. 3) ist keine Änderung der Stratigraphie erkennbar. - Zu der Grabungstätigkeit F. Miltners und der Kritik an seiner raschen Arbeitsmethode s. U. Quatember, Zur Grabungstätigkeit Franz Miltners an der Kuretenstraße, in: B. Brandt - V. Gassner - S. Ladstätter (Hrsg.), Synergia. Festschrift Friedrich Krinzinger I (Wien 2005) 271-278 bes. 278.

${ }_{18}$ Miltner 1959, 309.

${ }_{19}$ Miltner 1958b, 100. 
Vorhof aufgestellten >Großen Artemis` persönlich verantwortlich ${ }^{20}$ : »Die Christin ließ die Statue wohl stürzen, doch die Trümmer blieben unberührt am alten Platze liegen, denn das Geheimnis mystischen Glaubens umwehte auch noch das gestürzte Bild der Gottheit.« Aus diesen Gründen hätte man es auch nicht gewagt, die Statue nach ihrem Sturz völlig zu zerschlagen oder zu Kalk zu verbrennen ${ }^{21}$.

Die Christin Scholastikia, die uns lediglich als Stifterin der Renovierung der nach ihr benannten Badeanlage am unteren Embolos bekannt ist (IvE 453), aber nirgends als christliche >Eiferin< dargestellt wird, lässt zwar Bauglieder des Prytaneions in der Therme versetzen, sie aber mit der Zerstörung des Prytaneions in Verbindung zu bringen, ist ebenfalls rein spekulativ. Dies umso mehr, als zwischen der Zerstörung und dem tatsächlichen Beginn der Beraubung der Ruine eine erhebliche zeitliche Diskrepanz liegt. So vermutete bereits W. Alzinger, dass die Zerstörung der Anlage zwar in theodosianische Zeit datieren würde ${ }^{22}$, die Beraubung des Gebäudes und die Verbauung ihrer Architekturglieder aber erst später, als von F. Miltner angenommen, erfolgt wäre oder sich über einen deutlich längeren Zeitraum erstreckt hätte ${ }^{23}$. Diese Vermutung wird durch die Analyse des architektonischen und archäologischen Befundes der >Kuretenhalle am unteren Embolos bestärkt, in der zahlreiche Architekturglieder des Prytaneions - insbesondere Säulentrommeln - sekundär verbaut waren ${ }^{24}$ : Die Errichtung dieser Halle erfolgte erst in der ersten Hälfte des 6. Jahrhunderts n. Chr.

Zur Frage, ob das Prytaneion überhaupt von Christen zerstört wurde oder nicht schon früher einer Naturkatastrophe, wie etwa der Erdbebenserie der zweiten Hälfte des 4. Jahrhunderts n. Chr., zum Opfer fiel und danach schlicht

${ }^{20}$ Miltner 1958b, 101.

${ }^{21}$ Auch Knibbe (Anm. 3) 165 f. bezeichnet die »endgültige Zerstörung « des schon zuvor durch Erdbeben beschädigten Prytaneions als »das Werk von fanatischen Christen«. - Gegen eine intentionelle Zerstörung durch Christen: Karwiese (Anm. 9) 129. 131 f.; Scherrer (Anm. 9) 19; Büyükkolanc1 - Yüğrük (Anm. 9) 88.

${ }^{22}$ Er folgt dabei Miltner (Anm. 3:1956-1958) Beibl. 35 f.

${ }^{23}$ Und zwar »etwa gleichzeitig mit der Zerstörung der Basilika (Basilike Stoa, Anm. d. Verf.) um 500 n. Chr.«; vgl. RE Suppl. XII (1970) 1646-1648 s. v. Ephesos: B. Archäologischer Teil (W. Alzinger); Alzinger 1972-1975, 299. - Der Zeitpunkt der endgültigen Zerstörung

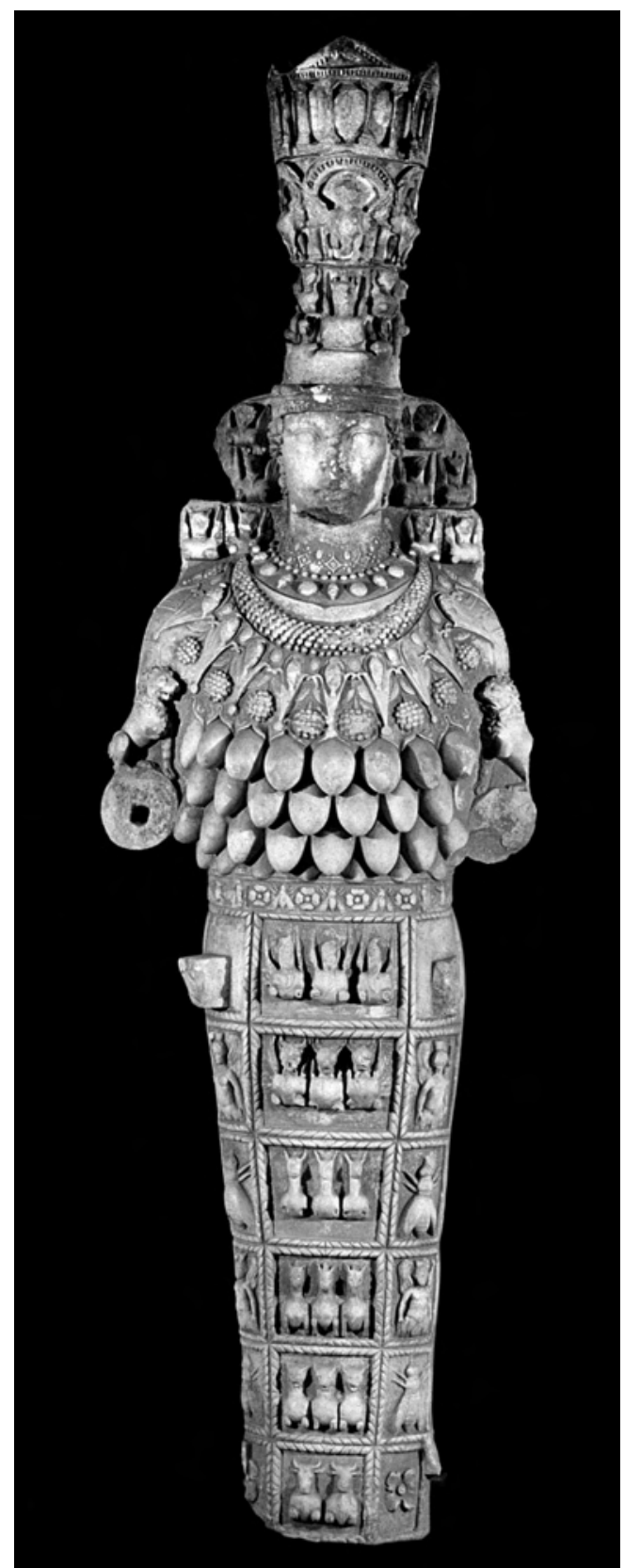

7 `Große Artemis` (Efes Müzesi Inv. 712) der Basilike Stoa ist bis dato nicht zufriedenstellend geklärt. Nach C. Lang-Auinger, Zusammenfassung, in: V. Mitsopoulos-Leon - C. Lang-Auinger (Hrsg.), Die Basilika am Staatsmarkt in Ephesos. 2. Teil: Funde klassischer bis römischer Zeit, FiE 9, 2, 3 (Wien 2007) 206 dürfte die Nutzung der Basilika über die Zeit »um $500 \mathrm{n}$. Chr.« hinausgehen: »Die Glasfunde, ein Teil der Lampen und etwa die Hälfte der Münzfunde belegen innerhalb der Basilika Baumaßnahmen bis in die Spätantike und eine Nutzung bis in das frühe 7. Jh. n. Chr. Bauliche Veränderungen innerhalb der Basilika lassen sich in diesem langen Nutzungszeitraum nur schwer fassen, auffallend sind jedoch die spätantiken Funde.«; vgl. auch V. Mitsopoulos-Leon, Die Basilika am Staatsmarkt in Ephesos. Kleinfunde. 1. Teil: Keramik hellenistischer und römischer Zeit, FiE 9, 2, 2 (Wien 1991) 13.

${ }^{24}$ s. dazu H. Thür, Die spätantike Bauphase der Kuretenstraße, in: R. Pillinger - O. Kresten - F. Krinzinger - E. Russo (Hrsg.), Efeso paleocristiana e bizantina - Frühchristliches und byzantinisches Ephesos, AForsch 3 = DenkschrWien 282 (1999) 115 f.; H. Thür - W. Pietsch, Prozessionsstraße (Via Sacra) im Bereich Kuretenstraße/Heroa/Hadrianstor, ÖJh 66, 1997, Grabungen 1996, 6-12; Thür (Anm. 9) 264 Anm. 36; A. Waldner, Keramische Evidenzen zur Baugeschichte des unteren Embolos von Ephesos (Diss. Universität Wien 2009) 164-167. 207 f. 
nicht wieder aufgebaut wurde, bleiben die aktuellen Forschungen zur Baugeschichte des Gebäudes abzuwarten $^{25}$. Spätestens mit dem kaiserlichen Verbot heidnischer Kultausübung durch Theodosius I. in den Jahren 391/392 n. Chr. ${ }^{26}$ war das Prytaneion jedenfalls wesentlicher Funktionen - insbesondere der kultischen enthoben $^{27}$.

\section{Ergebnis}

Nach der Darstellung der Fundsituation und Fundumstände der kaiserzeitlichen Artemis Ephesia-Statuen aus dem Prytaneion von Ephesos zeigt sich folgendes Bild:

Für die Statuen der >Großen Artemis und der >Kleinen Artemis $<$ konnte kein wie auch immer geartetes Argument für eine `kultische Bestattung`gefunden werden. Auch der Ausgräber, F. Miltner, äußert sich an keiner Stelle in diese Richtung. Diese Vermutung wird erst in der Sekundärliteratur getätigt, ohne entsprechende neue Belege beizubringen. Aber auch eine rituelle Deponierung der \Schönen Artemis $\measuredangle$ der der Ausgräber eine >kultische Bestattung \attestierte, ist nicht zu beweisen. Dieser Interpretation widersprechen sowohl die Fundumstände als auch der stratigraphische Befund. Folgende Gründe können dafür ins Treffen geführt werden:

- Die Grabungen im Prytaneion waren zum Zeitpunkt der Auffindung der Statue ohne archäologische Betreuung.

- Die erste Beurteilung der Fundsituation durch den Ausgräber lässt die Deponierungssituation noch offen. Erst im Vorbericht zu den Grabungen im Prytaneion wird eine Bestattung als »eindeutig« bezeichnet.

- Die Fragmente der \Schönen Artemis` werden von F. Miltner (1956) und W. Alzinger (1961) auf, aber auch unter dem als >Artemisniveau bezeichneten Nutzungshorizont, ja sogar in anderen Räumen gefunden. Dies und die Unvollständigkeit der Statue widersprechen der Vorstellung, die Statue wäre von den »letzten Anhängern" der Göttin bestattet worden, um sie vor christlichen Eiferern zu bewahren. Während Teile der Statue also vergraben waren, lag der Großteil der Statue auf dem Nutzungshorizont. Wesentliche Teile der Statue waren demnach nicht vergraben oder gar bestattet, sondern aus uns unbekannten Gründen auf dem >Artemisniveau zu liegen gekommen. Vergraben und bestattet hätte die Statue nur von einem noch jüngeren und höher gelegenen Nutzungsniveau aus werden können. Bei der Anlage der byzantinischen Nutzungsniveaus auf den Zerstörungsstraten des Prytaneions war das Gebäude allerdings schon devastiert und der gesamte Komplex mit Schutt bedeckt; auch die Statue wird zu diesem Zeitpunkt kaum mehr sichtbar auf ihrem ursprünglichen Platz gestanden sein. Es wäre somit neben der unvollständigen Deponierungssituation auch die zeitliche Diskrepanz zwischen den Deponierungsvorgängen zu argumentieren.

- Die Zerstörung des Gebäudes durch Christen ist nicht gesichert. Ein entsprechendes Bedrohungsszenario, das eine Bestattung der Statue zum Schutz vor dem sheiligen Eifer` der Christen begründen würde, ist folglich nicht belegt.

- Nicht schlüssig wäre schließlich die selektive Vorgehensweise der »letzten Anhänger« der Göttin, die nur eine der zumindest vier vorhandenen Artemis Ephesia-Statuen bestatteten, die anderen aber in ihrer Sturz- oder Deponierungslage zurückließen.

\footnotetext{
${ }^{25}$ So bereits Karwiese (Anm. 9) 131 f.; Scherrer (Anm. 9) 19. Zu dieser Erdbebenserie s. S. Ladstätter, Die Chronologie des Hanghauses 2, in: F. Krinzinger (Hrsg.), Das Hanghaus 2 von Ephesos. Studien zu Baugeschichte und Chronologie, AForsch 7 = DenkschrWien 302 (Wien 2002) 23-26. 29-31.

${ }^{26}$ Cod. Theod. 16, 10, $10 \mathrm{f}$.

${ }^{27} \mathrm{Zu}$ den im Prytaneion angesiedelten Kulten s. Knibbe (Anm. 3) 101-105. Neben den Hauptkulten der Hestia Boulaia und der Artemis - das Prytaneion fungierte gleichsam als Dependance des Artemisions innerhalb der Stadt - sind zu nennen: Demeter Karpophoros und ihre Tochter Kore, Sosipolis, Apollon Klarios, Apollon Manteios, Theos Kinnaios, Tyche und das personifizierte Heilige Feuer. - Zur Bedeutung des Prytaneions als Kultstätte der Artemis im Rahmen der jährlichen Prozession s. D. Knibbe - G. Langmann, Via Sacra Ephesiaca I, BerMatÖAI 3 (Wien 1993) 11. 21; Knibbe (Anm. 8$) 143$ f. 146 Anm. 8; P. Scherrer, Augustus, die Mission des Vedius Pollio und die Artemis Ephesia, ÖJh 60, 1990, $91 \mathrm{f}$.
} 
Ohne das Prinzip irreversibler Deponierungen in kultischem Kontext generell in Frage zu stellen, muss für das konkrete Beispiel gefolgert werden: Die immer wieder postulierte Vorstellung kultischer oder ritueller Bestattungen der Artemis Ephesia-Statuen im Prytaneion von Ephesos ist aus genannten Gründen nicht nur in Zweifel zu ziehen, sondern abzulehnen, da sie durch nichts zu belegen ist. Die Statuen wurden entweder in Sturzlage gefunden oder in der Position ihrer endgültigen Deponierung, deren Gründe wir nicht kennen.

Mag. Dr. Martin Steskal

Österreichisches Archäologisches Institut, Franz Klein-Gasse 1, A-1190 Wien

E-Mail:martin.steskal@oeai.at

Abbildungsnachweis: Abb. 1: Photo: M. Steskal; Abb. 2: Graphik L. Zabrana, P. Mayrhofer, M. Steskal; Abb. 3: Photo (C) ÖAI; Abb. 4. 5. 7: Photo N. Gail, C ÖAI; Abb. 6: Photo (C) ÖAI. 
